ISSN: 2582-0435

Meta Analysis | Vol 4 Iss S9

\title{
Innovative Medicines \& Medical Practice during COVID at MENA Region
}

\author{
Abdalla Abotaleb \\ Department of Cancer, World Health Organisation, Egypt \\ Correspondence should be addressed to Abdalla Abotaleb, thepharmacist7777@gmail.com
}

Received: November 26, 2020; Accepted: December 29, 2020; Published: January 05, 2021

\section{KEYWORDS}

Policy analysis; Innovative treatments; Medical practice; COVID -19

\section{OBJECTIVES}

Medical practice during COVID -19 pandemic faced many challenges one of the major challenges is adaptation of treatment protocols for achieving following objectives.

Managing system capacity, enhancement of patient's outcomes (quality of life - economic value - clinical effectiveness). And utilizing the resources during this hard times.

Previous objectives raised many questions about nature of treatment protocols and policies should be implemented during (COVID -19) pandemic and how innovative medicines can achieve the previous objectives. At MENA countries (Algeria, Morocco, Egypt, Lebanon, KSA, UAE and Turkey) at four specialties (Oncology, cardio metabolic, ophthalmology, rare diseases) [1-12].

\section{METHOD}

Integration analysis between International guidelines like (ESMO, ASCO, WHO, ESC, NHS) and local guidelines. Local survives with local physicians, health authorities at previous countries plus quality of life questionnaires for the patients were conducted. Cost analysis from health systems for all health care expenditures.

One way sensitivity analysis was conducted for all parameters affecting on practice adaptation to ensure validity and accuracy.

\section{RESULT}

Innovative medicines had positive impact on enhancement of patient's outcomes and resources utilization via implementation efficient. Periodization criteria as following:

\section{Oncology Practice}

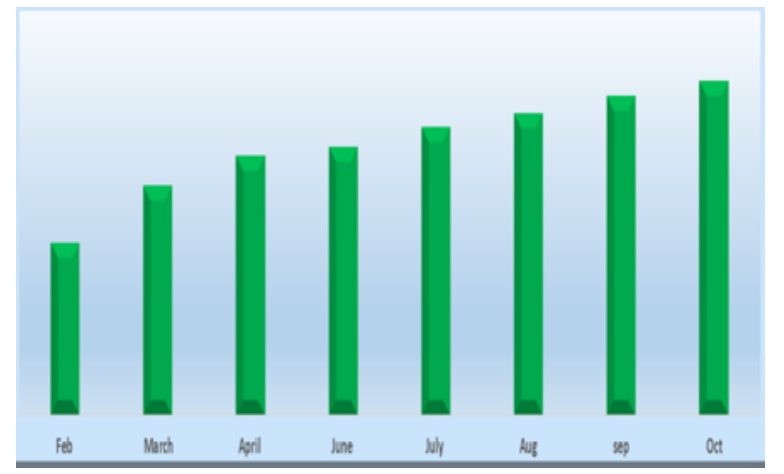

Figure 1: Quality of life for HCC patients using innovative treatments. 


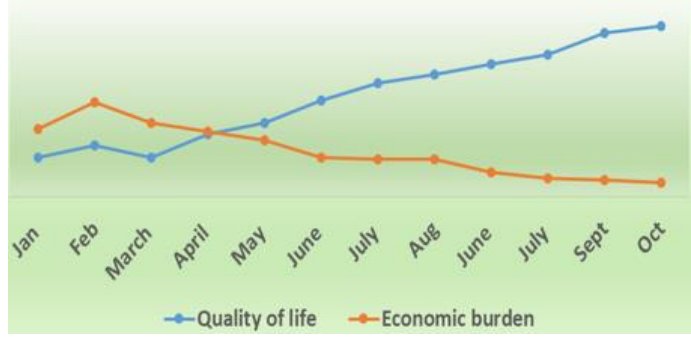

Figure 2: Sorafenib analysis for KSA.

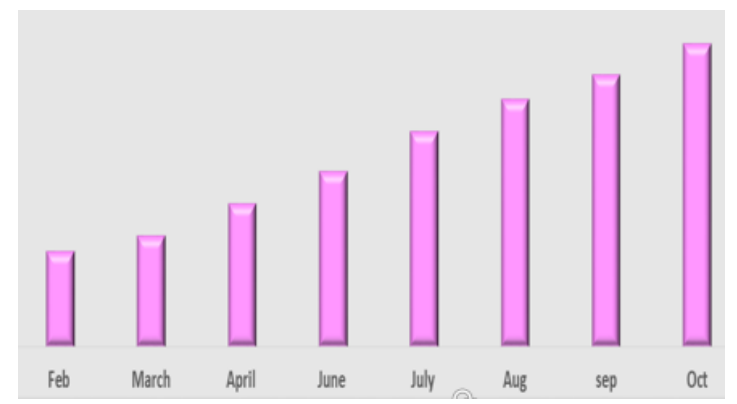

Figure 3: Quality of life for breast cancer using innovative treatments.

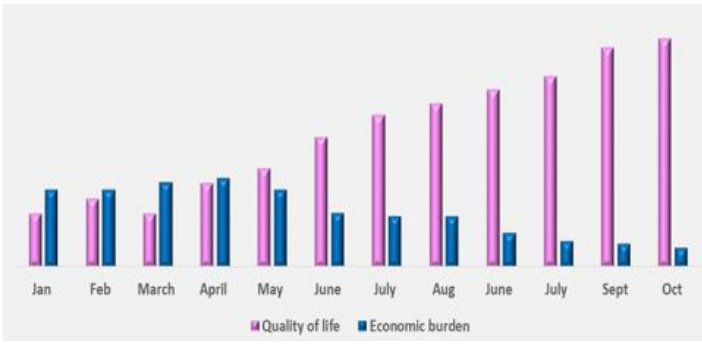

Figure 4: Analysis of Trastuzumab SC at MENA.

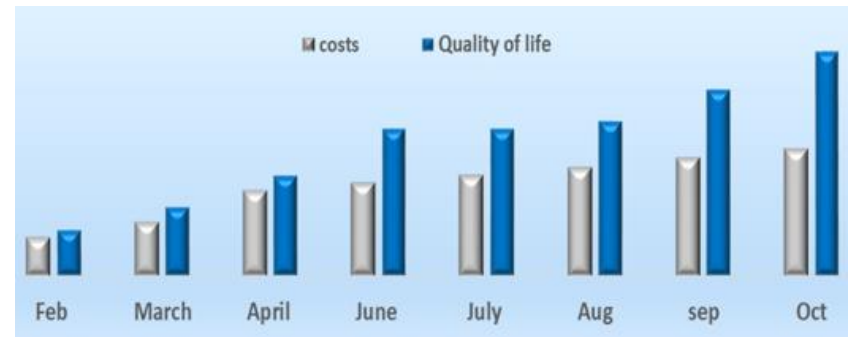

Figure 5: Costs combined with quality of life for CRC patients.

\section{Ophthalmology Practice}
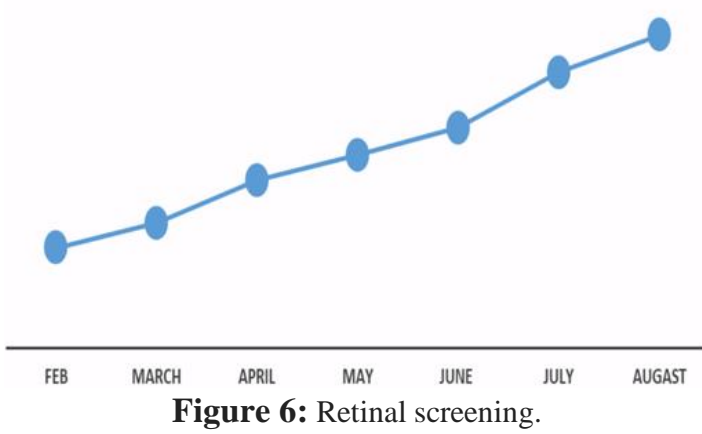

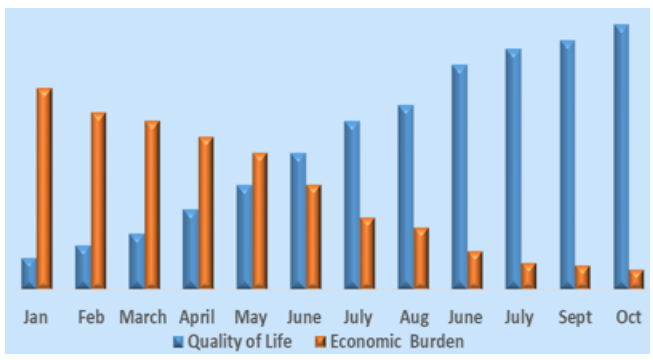

Figure 7: Qulaity of life combined with economic burden for DME patients.

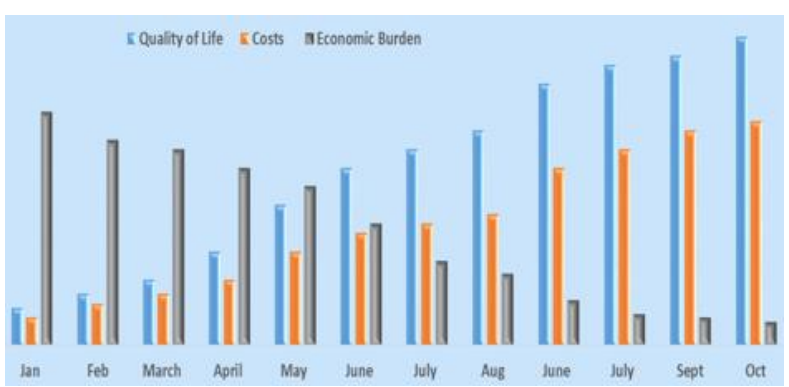

Figure 8: DME outcome elements.

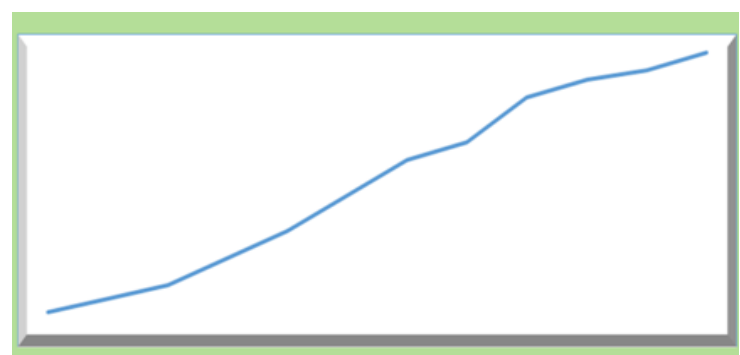

Jan Feb March April May June July Aug June July Sept Oct

Figure 9: Quality of life for patients with Aflibercept.

\section{Cardio Metabolic Practice}

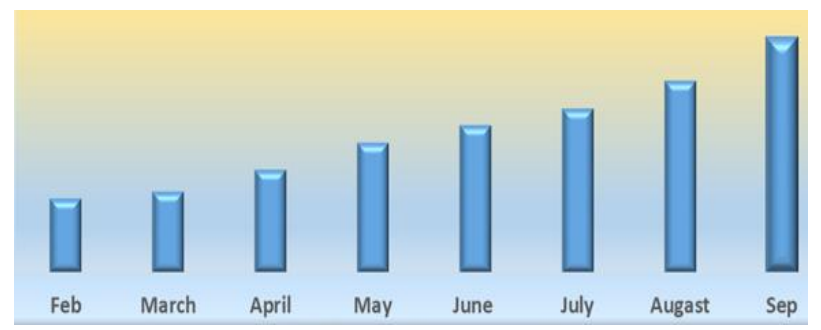

Figure 10: Costs of diabetes \& complications management during COVID-19 at MENA.

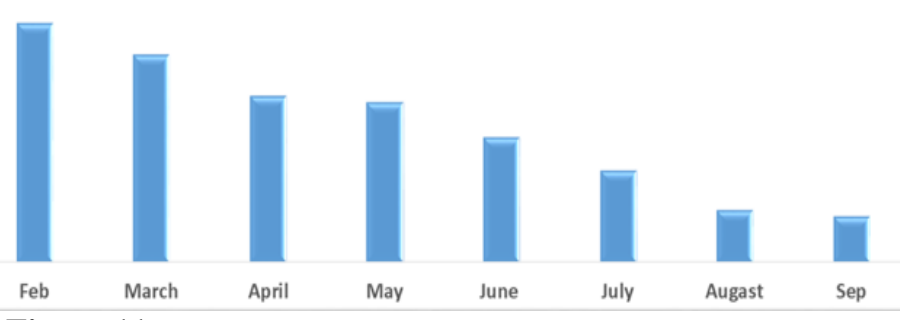

Figure 11: Costs of prediabetes management during COVID19 at MENA. 


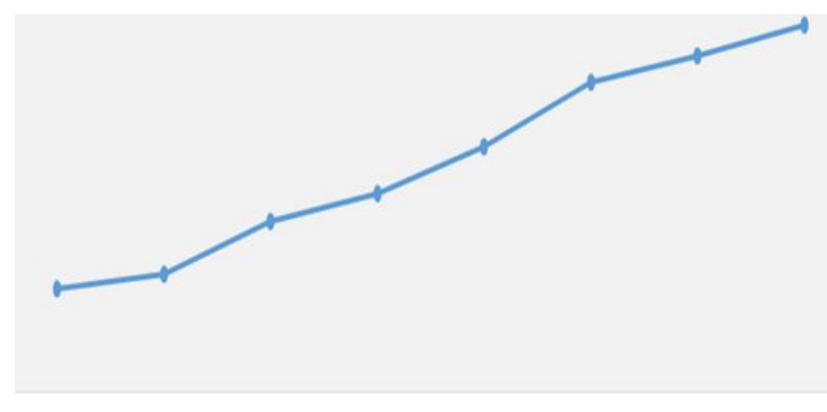

Feb March April May June July Augast Sep

Figure 12: Quality of life with Metformin.

\section{Rare Diseases}

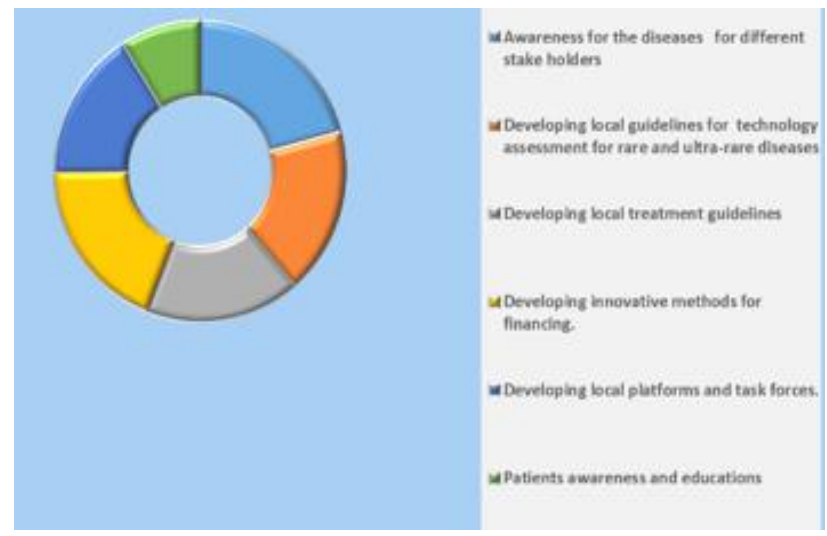

Figure 13: Results for rare diseases.

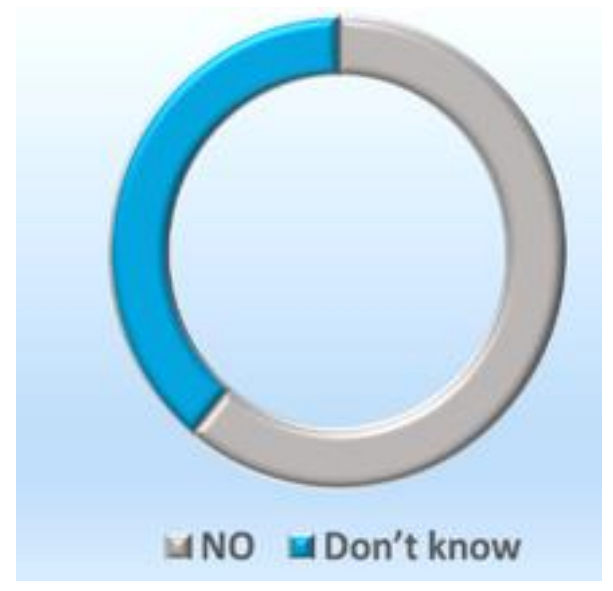

Figure 14: Pyers knowledge about WTP for rare diseases.

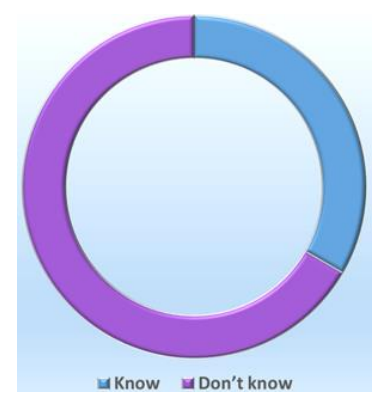

Figure 15: Pyers knowledge for pricing processes of rare diseases products.

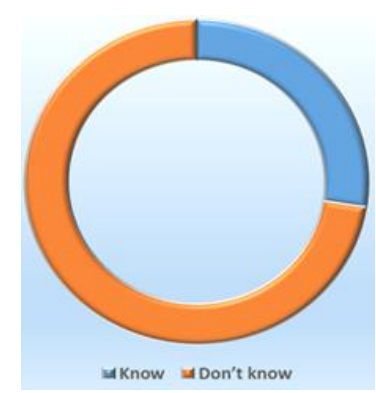

Figure 16: Payers knowledge for definition of rare \& ultra rare diseases.

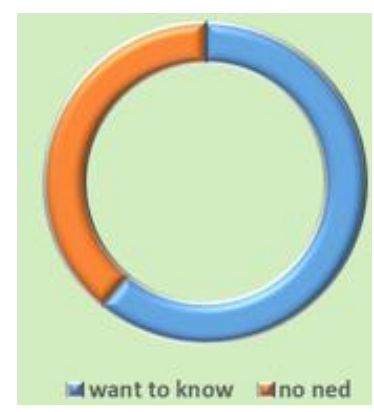

Figure 17: Payers willing to know about rare \& ultra rare diseases health policies.

\section{CONCLUSION}

Innovative medicines protocols changed the medical practice during COVID-19 through dynamic \& efficient prioritization criteria which leaded to patient's outcomes (quality of life - economic value - clinical effectiveness) enhancement plus resources utilization.

\section{CONFLICT OF INTEREST}

No conflict of interest.

\section{REFERENCES}

1. World Health Organization (2020) Novel coronavirus (COVID-19) situation.

2. (2020) Clinical guide for the management of non-coronavirus patients requiring acute treatment: Cancer.

3. (2020) ESMO clinical guidelines for the management of non-coronavirus patients. 
http://www.tridhascholars.org | June-2021

4. Wu Z, McGoogan JM (2020) Characteristics of and important lessons from the coronavirus disease 2019 (COVID-19) outbreak in China: Summary of a report of 72314 cases from the Chinese center for disease control and prevention. JAMA 323(13): 1239-1242.

5. ESMO (2020) The ESMO-MCBS score card.

6. Li B, Yang J, Zhao F, et al. (2020) Prevalence and impact of cardiovascular metabolic diseases on COVID-19 in China. Clinical Research in Cardiology 109(5): 531-538.

7. Long B, Brady WJ, Koyfman A, et al. (2020) Cardiovascular complications in COVID-19. The American Journal of Emergency Medicine.

8. Garcia S, Albaghdadi MS, Meraj PM, et al. (2020) Reduction in ST-segment elevation cardiac catheterization laboratory activations in the United States during COVID-19 pandemic. Journal of the American College of Cardiology 75(22): 2871-2872.

9. Tam CCF, Cheung KS, Lam S, et al. (2020) Impact of coronavirus disease 2019 (COVID-19) outbreak on ST-segmentelevation myocardial infarction care in Hong Kong, China. Circulation: Cardiovascular Quality and Outcomes 13(4): $\mathrm{e} 006631$.

10. Zhou F, Yu T, Du R, et al. (2020) Clinical course and risk factors for mortality of adult inpatients with COVID-19 in Wuhan, China: A retrospective cohort study. The Lancet 395(10229): 1054-1062.

11. Skulstad H, Cosyns B, Popescu BA, et al. (2020) COVID-19 pandemic and cardiac imaging: EACVI recommendations on precautions, indications, prioritization, and protection for patients and healthcare personnel. European Heart JournalCardiovascular Imaging 21(6): 592-598.

12. (2020) ESC guidance for the diagnosis and management of CV disease during the COVID-19 pandemic. 